\title{
Pemetrexed and Gemcitabine for Chemotherapy Refractory Colorectal Cancer-Results of a Phase II and Translational Research Study
}

\author{
Karen-Lise Garm Spindler ${ }^{1 \#}$, Niels Pallisgaard ${ }^{2}$, Rikke Fredslund Andersen², John Ploen ${ }^{1}$, \\ Anders Jakobsen ${ }^{1}$ \\ ${ }^{1}$ Department of Oncology, Vejle Hospital, Vejle, Denmark; ${ }^{2}$ Department of Biochemistry, Vejle Hospital, Vejle, Denmark. \\ Email: ${ }^{\#}$ Karen-Lise.Garm.Spindler@slb.regionsyddanmark.dk
}

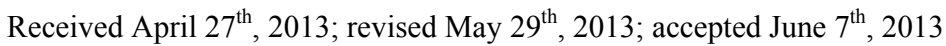

Copyright (C) 2013 Karen-Lise Garm Spindler et al. This is an open access article distributed under the Creative Commons Attribution License, which permits unrestricted use, distribution, and reproduction in any medium, provided the original work is properly cited.

\begin{abstract}
Introduction: We investigated the safety and efficacy of pemetrexed with gemcitabine in heavily pre-treated, chemotherapy refractory, KRAS mutated colorectal cancer (mCRC) and the prognostic value of quantitative levels of cell free DNA (cfDNA) in plasma. Methods: Inclusion criteria comprised; histopathologically verified, KRAS mutant, chemotherapy resistant $\mathrm{mCRC}$, adequate organ function and performance status. Patients received pemetrexed (initially 500 $\left.\mathrm{mg} / \mathrm{m}^{2} \mathrm{q} 3 \mathrm{w}\right)+$ gemcitabine $\left(1250 \mathrm{mg} / \mathrm{m}^{2}\right.$ days 1 and 8$)$ until progression or unacceptable toxicity. RECIST version 1.1, NCI-CTCAE version 4.0 and Kaplan-Meier statistics were used for endpoint evaluation. Cell free DNA was quantified from pre-treatment EDTA plasma-samples by an in-house qPCR. Results: Forty patients were included. The median number of cycles was 3 (range 0 - 12). Thirty-six percent obtained disease stabilisation, but no objective response was observed. Median PFS and OS were 2.8 (range 2.1 - 4.0) and 5.4 (range 4.3 - 7.0) months, respectively. Adverse events caused immediate discontinuation of treatment or delay of the next cycle and consequently discontinuation in 5 patients. Translational research revealed a shorter PFS and OS with increasing levels of cfDNA. The median PFS in patients with cfDNA levels above the 75 percentile was 2 months compared to 4 months in the remaining patients, HR 3.23 (1.05 9.89), $\mathrm{p}=0.0008$. The median OS was 3 and 6 months, respectively, HR 2.9 (95\%CI $0.98-8.34)$. Cox regression analysis confirmed that cfDNA remained a significantly independent prognostic factor for both PFS and OS. Conclusion: Pemetrexed and gemcitabine did not prove sufficient benefit and unacceptable toxicity was observed. The potential value of cfDNA should be investigated further.
\end{abstract}

Keywords: Metastatic Colorectal Cancer; KRAS; cfDNA; Plasma; Pemetrexed; Gemcitabine

\section{Introduction}

A substantial proportion of the colorectal cancer patients who fail on standard chemotherapy is in good performance status and eligible for other treatment options. KRAS status is decisive for effect of combination therapy with epidermal growth factor receptor inhibitors, since benefit is confined to KRAS wild type patients. Thus, chemotherapy refractory patients with KRAS mutant disease have no established treatment options, and experimental trials are warranted to identify new possible effective regimens for this group of patients.

Pemetrexed is a methotrexate analogue and a folate

"The authors declare no conflicts of interest/have no disclosures.
${ }^{*}$ Corresponding author. antimetabolite inhibiting folate dependent enzymatic pathways essential for cell replication. The mechanisms of cytotoxicity are partly thymidylate synthase (TS) inhibition, partly targeting other enzymes such as the dihydrofolate reductase (DHFR) and glycinamide ribonucleotide formyl transferase (GARFT) involved in purine synthesis [1]. Pemetrexed has demonstrated efficacy in preclinical models of colorectal cancer cell lines [2] and response rates of 15.4 and 17.2 in first line monotherapy studies $[3,4]$. It has also been evaluated in combination with both irinotecan and oxaliplatin with non-inferior response rates and acceptable toxicity compared to standard treatment of metastatic disease [5-7] Preliminary data suggest that $5 \mathrm{FU}$ resistant tumours might respond to 
pemetrexed based treatment; however, this aspect has not been sufficiently investigated [8-10].

Gemcitabine has shown effect in colorectal cancer, but minimal efficacy as a single agent in fluoropyrimidine resistant disease. However, a recent data analysis of 144 patients with refractory $\mathrm{mCRC}$, who were treated with combinations of gemcitabine and fluoropyrimidines, revealed a RR above $30 \%$. Toxicity was manageable, except from regimens including bolus 5FU [11].

An in vitro-synergistic effect between pemetrexed and gemcitabine has been demonstrated in different tumour types, including the colorectal cancer HT29, LoVo, WiDr and LRWZ cell lines [12,13] Pemetrexed accumulates cells in the S-phase, which helps gemcitabine incorporation and apoptosis, and pemetrexed enhances upregulation of $\mathrm{cCK}$, which phosphorylates gemcitabine and renders the drug active. Hereby pemetrexed has the potential to sensitize cells to the cytotoxic activity of gemcitabine. Different dosing schedules of pemetrexed and gemcitabine combinations have been evaluated and [14] the most efficient regimen with the lowest degree of toxicity has been established by a direct comparison made by Ma et al. in NSCLC patients [15]. Consequently, the combination is an established treatment option in NSCLC and both pre-clinical and clinical data support the investigation in chemotherapy refractory CRC.

Translational research is a prerequisite for improvement of patient selection for relevant therapy and for avoiding treatment with no benefit and harmful toxicity. We have previously shown that circulating free DNA (cfDNA) can be detected in the peripheral blood and that the level of cfDNA alleles was correlated to outcome of third line treatment in a cohort of patients with heavily pre-treated mCRC [16]. Prospective collection of blood samples for TR studies is therefore highly relevant in this setting.

The present study aimed to investigate the efficacy and safety of the combination of pemetrexed and gemcitabine in heavily pre-treated, chemotherapy resistant, KRAS mutated colorectal cancer. In parallel, translational research studies were conducted focusing on quantitative measurement of cfDNA in the blood.

\section{Materials and Methods}

\subsection{Patient Selection}

Patients with histologically verified colorectal cancer with KRAS mutations, who had failed standard chemotherapy with fluoropyrimidines, oxaliplatin and irinotecan were eligible for the study. Other requirements included measurable disease according to RECIST; age $\geq$ 18 years; Eastern Cooperation Oncology Group performance status of $0-2$; adequate bone marrow and biochemical function and acceptance of sampling for trans- lational research.

Patients were excluded who had clinical signs of or known brain metastasis; other clinically significant concurrent illness at the discretion of the investigator; pregnant or breastfeeding patients; other experimental treatment within 30 days prior to study entry; planned radiation of target lesions and concurrent vaccination against yellow fever.

All patients provided signed informed consent before study entry and the protocol was approved by the Danish Medicines Agency and The Regional Scientific Ethical Committee of Southern Denmark. The study was conducted in accordance with the Good Clinical Practice guidelines as issued by the International Conference on Harmonization and the Declaration of Helsinki.

\subsection{Treatment}

Patients initially received intravenous pemetrexed 500 $\mathrm{mg} / \mathrm{m}^{2} \mathrm{q} 3 \mathrm{w}$ combined with gemcitabine $1250 \mathrm{mg} / \mathrm{m}^{2}$ days 1 and 8 . A dosage cap at $2 \mathrm{~m}^{2}$ was required and a $75 \%$ dosing schedule used in patients who had previously received chemotherapy in reduced dosing levels. Out of safety concerns after the first 25 patients the starting dose level was reduced to $80 \%$ of the primarily planned schedule (pemetrexed $400 \mathrm{mg} / \mathrm{m}^{2}$ and gemcitabine $1000 \mathrm{mg} / \mathrm{m}^{2}$ ). Premedication with oral prednisolone $25 \mathrm{mg}$ was given twice the day before, on the treatment day and the day after all doses of pemetrexed. Antiemetics according to local guidelines supplemented with $350 \mu \mathrm{g}$ oral folic acid daily and $1000 \mu \mathrm{g}$ vitamin B12 intramusculary $\mathrm{q} 9 \mathrm{w}$ were required.

CT scans of chest and abdomen were used for evaluation of response according to RECIST (version 1.1) and performed less than 3 weeks prior to first treatment given and every 9 weeks during treatment. Follow-up scans were performed every three months in patients who stopped treatment on stable disease and continued until first sign of progression. The NCI-CTCAE version 4.0 was used to assess toxicity and recorded at every visit for treatment until resolved. During combination therapy any dose reduction was applied to both drugs regardless of the type of toxicity.

Patients were treated until progression, unacceptable toxicity, prolonged treatment pause or withdrawal of consent.

\subsection{Trial Design and Statistics}

The trial was designed as a single arm, phase II study according to Simon's two stage designs. A response rate higher than $25 \%$ was considered clinically relevant with the perspective of further investigations, whereas less than $10 \%$ was deemed unacceptable. $\alpha$ was set to 0.05 and $\beta$ to 0.1 (a power of $90 \%$ ). Consequently, the initially 
planned sample size for the first step was 31 patients. If $\geq 4$ patients achieved an objective response to treatment, enrolment would be extended by another 24 patients to a maximum of 55 patients.

The primary endpoint was response according to RECIST version 1.1. Secondary endpoints included tolerability graded according to NCI-CTCAE version 4.0, progression free survival (PFS) calculated from date of first treatment until date of progression or death from any cause, and overall survival (OS) as defined from date of first treatment until death from any cause.

The association between marker status and objective response, baseline characteristics and toxicity rates was determined by two-sided t-tests or $\chi^{2}$-test. The KaplanMeier method was used to estimate PFS and OS. A multivariate Cox regression analysis was performed to examine whether the different variables were associated with reduced survival. P-values referred to two-tailed tests and were considered significant when $p \leq 0.05$. Statistics were carried out using the NCSS statistical software 2007 v.07.1.5 (NCSS Statistical Software, Utah 84,037, USA, www.ncss.com).

\subsection{Biomarker Collection and Analysis}

Archived tumor tissue was obtained and blood samples for translational research were drawn at baseline and prior to each cycle. KRAS tumor status was determined prior to treatment. Plasma samples were analysed for cfDNA by an in-house qPCR method as previously published [16].

\section{Results}

\subsection{Patients}

During the period from April 2010 to Aug 2011, 40 patients were included in the study comprising 19 female and 21 male patients. Seventy three percent of the patients had been diagnosed with primary, disseminated disease and the median time from primary diagnosis until inclusion in the study was 23 months (range 5 - 73 months). All patients had progressed on standard cytotoxic treatment and the majority $(73 \%)$ had also received anti-angiogenesis therapy. The pre-treatment characteristics are presented in Table 1.

Table 1. Pre-treatment characteristics $(\mathrm{N}=40)$ and correlation to cfDNA.

\begin{tabular}{|c|c|c|c|c|}
\hline Characteristic & Number & $\%^{* *}$ & $\begin{array}{l}\text { cfDNA alleles/ml plasma } \\
\text { Median ( } 95 \% \mathrm{CI})\end{array}$ & p-value \\
\hline Age median years (range) & $66(40-80)$ & & & \\
\hline$>$ age 66 & 19 & 52 & $8011(9070-25,917)$ & \\
\hline \multicolumn{5}{|l|}{ Gender } \\
\hline Male & 21 & 53 & $11,285(6848-18,011)$ & \\
\hline \multicolumn{5}{|l|}{ ECOG performance status } \\
\hline 0 & 19 & 48 & $15,446(6997-27,794)$ & \\
\hline 1 & 18 & 45 & $25,028(8611-43,190)$ & $\mathrm{p}>0.05$ \\
\hline 2 & 3 & 8 & $325,692^{*}$ (NA) & \\
\hline \multicolumn{5}{|l|}{ Site of primary tumor } \\
\hline Rectum & 16 & 40 & $28,325(9070-44,104)$ & $\mathrm{p}>0.05$ \\
\hline Colon & 23 & 58 & $15,486(6997-25,917)$ & \\
\hline NA & 1 & 3 & NA & \\
\hline \multicolumn{5}{|l|}{ Sites of metastatic disease } \\
\hline Liver metastasis present & 31 & 78 & $25,047(15,486-41,118)$ & $p=0.03$ \\
\hline No liver metastasis & 9 & 23 & $7567(4677-32,457)$ & \\
\hline Lung metastasis present & 30 & 75 & $21,510(12,062-32,457)$ & $\mathrm{p}>0.05$ \\
\hline No lung metastasis & 10 & 25 & $19,815(6073-44,104)$ & \\
\hline \multicolumn{5}{|l|}{ KRAS status in plasma } \\
\hline Mutation detected & 33 & 83 & $25,008(12,400-32,457)$ & $\mathrm{p}>0.05$ \\
\hline
\end{tabular}

* only 3 observations, and therefore (by t-test of PS 0 vs $3 ; \mathrm{p}=0.05) ;{ }^{* *}$ rounding of numbers. 
Thirty-nine of the forty patients commenced treatment as planned and the median number of cycles was 3 (range 0 - 12). The reasons for discontinuation were; disease progression in the majority of patients $(26(67 \%))$, toxicity $(1(2 \%))$, postponed course due to adverse events (4 $(10 \%))$ and patient's wish $(8(21 \%))$. After the interim analysis showed unsatisfactory response rates the patients still on treatment were informed and consequently discontinued protocol treatment. A total of 25 patients received $\geq 3$ cycles and were available for radiological response evaluation. An additional three patients had an evaluation scan after the second cycle and showed progressive disease and were consequently included in the interim analysis for efficacy assessment.

\subsection{Efficacy}

A total of 10 patients $(10 / 28=36 \%)$ obtained disease stabilisation, but no objective responses were observed, and 18 patients progressed before or at the first assigned response evaluation. The study was designed to include 31 evaluable patients in the interim analysis and continue into step 2 if four or more patients responded. Since none of the first 28 patients showed objective response after 3 cycles, accrual for the second step of the study was cancelled. At time of analysis seven patients were still alive, and the median PFS and OS were 2.8 (range 2.1 - 4.0) and 5.4 (range 4.3 - 7.0) months, respectively (Figure 1).

\subsection{Safety}

All AEs and SAEs were recorded including disease related events. There were no treatment related deaths or SUSARs reported, but a total of 35 SAEs were recorded as possibly treatment related. The majority of the events were episodes of pyrexia, which, due to standard procedures, caused hospital admission to exclude febrile neutropenia. However, initially the high frequency of complications led to modification of the protocol and a primary dose reduction. Toxicity resulted in immediate discontinuation of treatment $(n=1)$ or postponement of the next planned cycle and consequently discontinuation of therapy for a total of five patients.

\subsection{Translational Research}

All patients had pre-treatment blood samples available for measurement of cfDNA in the plasma. The mean and median cfDNA levels were 99,200 (range 2210 $2,259,700)$ and $19,800(95 \%$ CI 11,300 - 27,800) alleles per $\mathrm{ml}$. The 25 th percentile was 7800 and the 75 th percentile 42,700 alleles per $\mathrm{ml}$. The level of cfDNA was investigated in relation to all pre-treatment characteristics as shown in Table 1. A significantly higher median cfDNA level was revealed in female patients $(29,650$

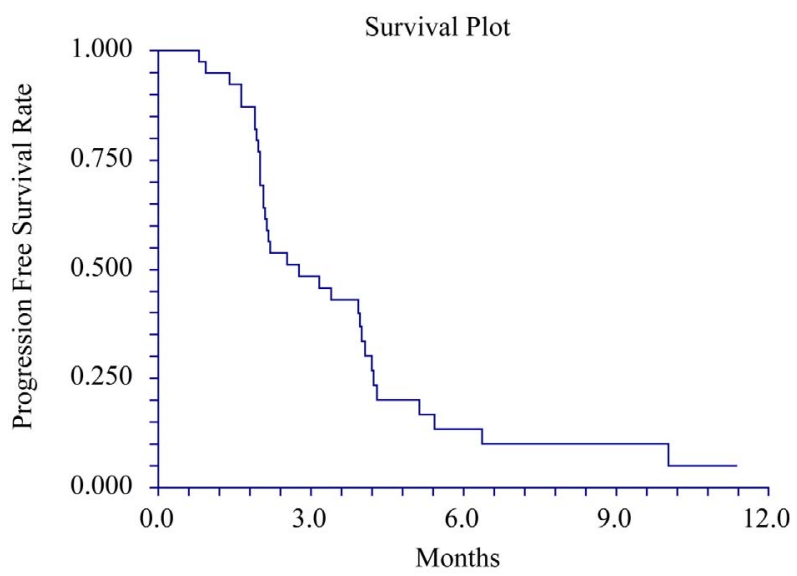

(a)

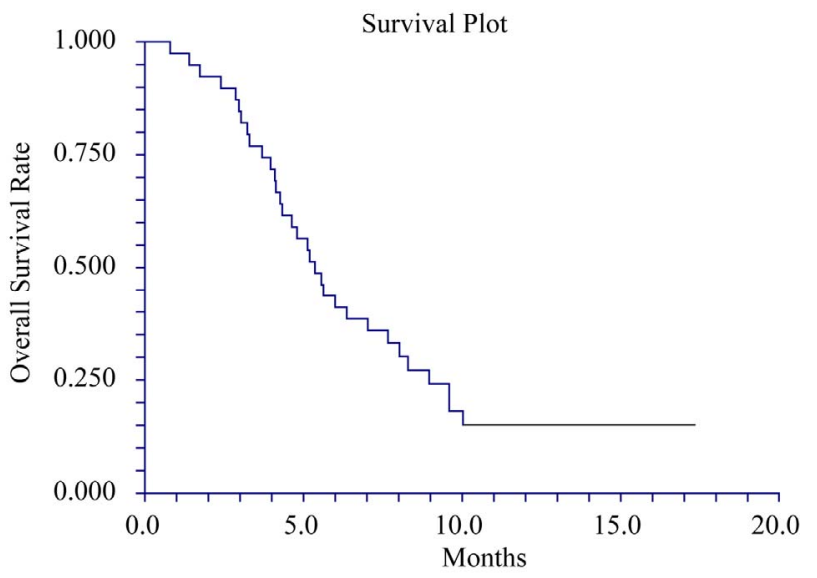

(b)

Figure 1. Kaplan Meier survival plots of PFS and OS in the total cohort of 40 patients.

alleles per ml) compared to male (median 11290 alleles per $\mathrm{ml}$, and in patients with liver metastasis compared to those with non-liver metastatic disease (median 25,000 and $7570, p=0.029$, respectively. There was a marginally higher cfDNA level in the three patients with poor PS (PS 2), compared to the remaining groups ( $p=0.049)$, and non-significant differences within the remaining parameters (data not shown).

Kaplan-Meier survival plots for PFS and OS revealed a shorter PFS and OS with increasing levels of pretreatment cfDNA when divided into quartiles of cfDNA levels. The median PFS in patients with the highest level of cfDNA was only 2 months compared to 4 months in the remaining patients, HR 3.23 (1.05 - 9.89), p = 0.0008. This translated into a significant difference in median overall survival of three and six months, respectively, HR 2.9 (95\%CI 0.98 - 8.34), (Figure 2). The number of events allowed for a multivariate cox regression analysis including the chosen variables age, gender, PS, presence of liver metastases, and plasma cfDNA divided into 
quartiles. Only cfDNA remained a significantly independent prognostic factor for both PFS and OS as presented in Table 2.

\section{Discussion}

Although a substantial fraction of the patients obtained stabilisation of the disease, no objective response was

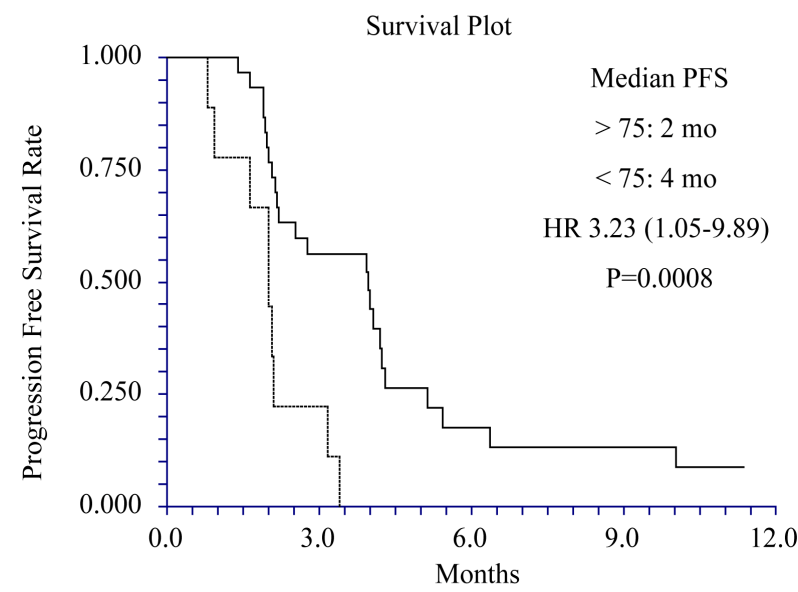

(a) observed and it must be concluded that, despite a preclinical as well as clinical rationale for combining pemetrexed and gemcitabine, we failed to demonstrate a clinically meaningful efficacy of this combination.

The search for effective treatment options for heavily pre-treated patients with chemotherapy refractory disease has taken considerable focus, but results have been disappointing. KRAS mutant, chemotherapy resistant disease

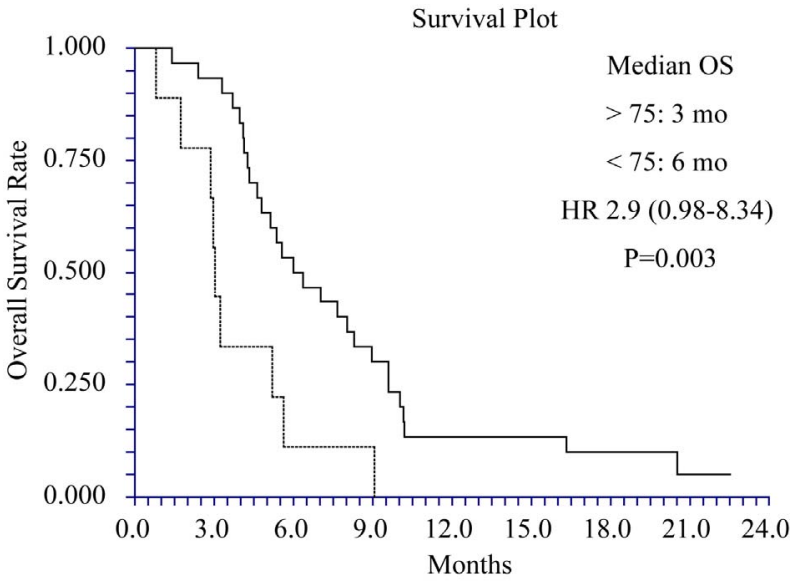

(b)

Figure 2. Kaplan Meier plots of the total cohort of patients dichotomised at the $\mathbf{7 5}$ percentile, which show increasing PFS and OS with decreasing pre-treatment plasma cfDNA levels. The dotted line represents patients with cfDNA levels above the 75 percentile and the full line the remaining patients.

Table 2. Multivariate cox regression analysis of PFS and OS.

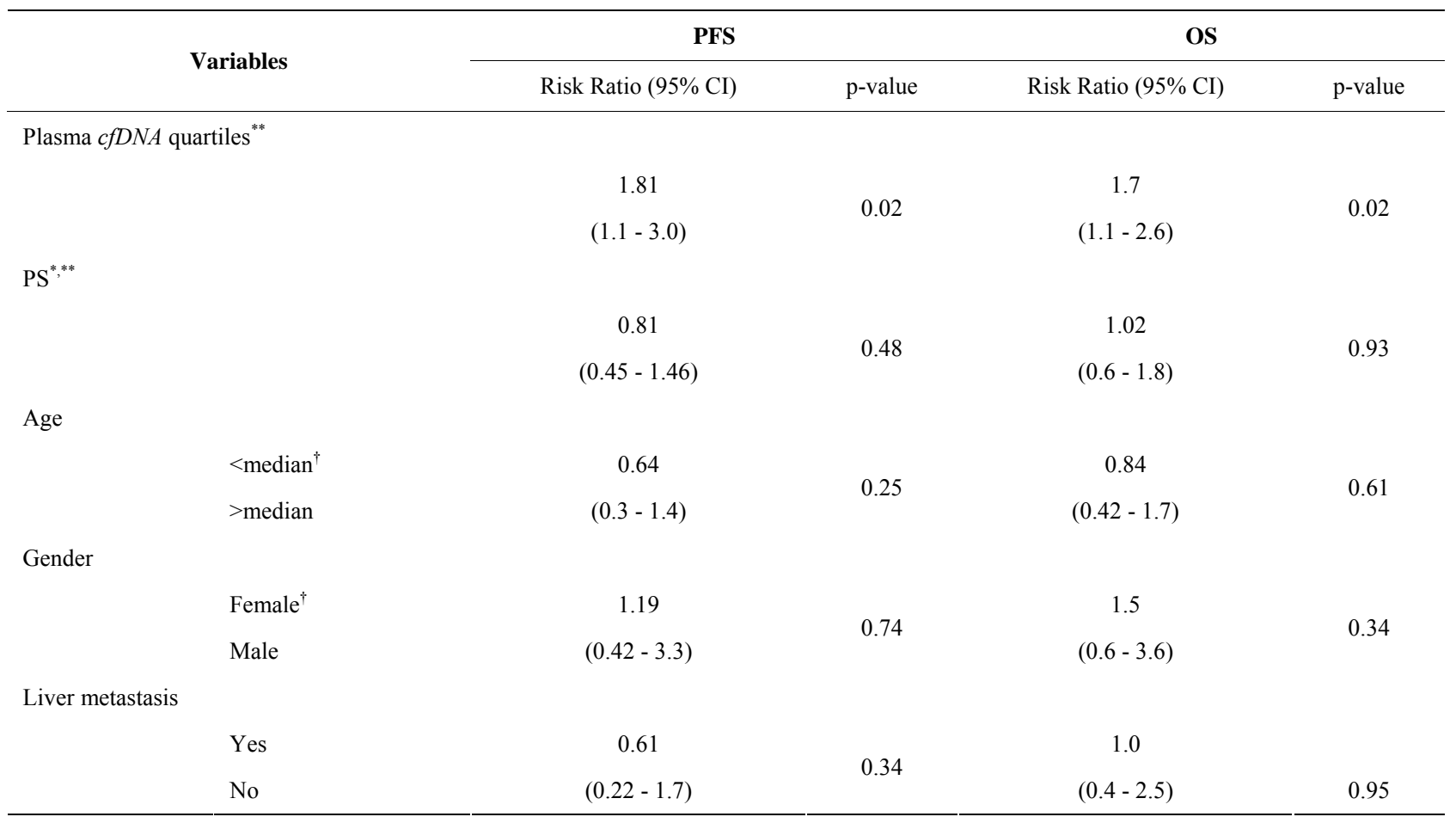

'reference group; ${ }^{*} \mathrm{PS}=$ Performance Status (ECOG); ${ }^{* *}$ entered as a continuous variable; The Risk Ratio refers to moving from the reference group to the other group or changing one step in parameters entered as continuous variables. 
therefore remains a major challenge to the treating physician and holds a poor prognosis. The median OS of our patients in this study was only 5.4 months, ranging from 4.3 - 7.0 months, and the PFS 2.8 months $(2.1-4.0)$. In comparison, a retrospective study investigating mitomycin C in 109 refractory mCRC patients showed OS of 4.5 months [17] and Vorinostat combined with 5-FU/LV was used in another study and produced a median PFS and OS of 2.4 and 6.5 months, respectively. Only one out of 43 patients achieved a partial response [18]. A Japanese study investigated salvage S-1 monotherapy in 23 patients who had failed on 5-FU, oxaliplatin and irinotecan and observed SD in $17 \%$ of the patients but no objective responses. The median PFS was 2.7 months (range 0.4 12.6) and OS 4.7 months (1.0 - 28.9) [19]. Thus, our data are comparable to the literature and unfortunately illustrate the rapidly progressive nature of disease at this stage. Results of new targeted agents are eagerly awaited, since traditional chemotherapeutic drugs have failed in this setting.

The present study has also underlined that although these patients are seemingly in good performance status with apparently healthy bone marrow function, a general exhaustion of the organ function renders these patients more at risk of toxicity. Results are definitely not comparable to chemotherapy naïve patients, who according to the literature showed significantly less toxicity from the present treatment regimen and we chose to reduce the starting dose. It is reasonable to conclude that despite a chance of disease stabilisation, the price paid in terms of hospital visits, admissions and need for surveillance is too high to justify active treatment of this group of patients. On the other hand, it must not be dismissed that upon careful selection a small subgroup could benefit from aggressive treatment in this setting. Therefore, reliable selection criteria should be investigated.

The present trial has enabled us to confirm the level of cfDNA alleles in the plasma to reflect disease behavior. As also demonstrated in two previous phase II studies by our group [16 and data in press], a high level of cfDNA was associated with a poor prognosis, and stabilisation of disease seemed confined to patients with low plasma levels. Consequently, it is likely that the level of cfDNA in the peripheral blood can serve as a selection criterion and that we hereby can avoid unnecessary toxicity and improve the clinical results. Prospective studies investigating this issue should therefore be designed.

In conclusion, pemetrexed and gemcitabine did not provide sufficient benefit in this setting and unacceptable toxicity was observed. However, despite the small sample size translational research has confirmed the clinical potential of measuring cfDNA levels prior to treatment and further studies on these aspects are highly relevant.

\section{REFERENCES}

[1] H. S. Hochster, "The Role of Pemetrexed in the Treatment of Gastrointestinal Malignancy," Clinical Colorectal Cancer, Vol. 4, No. 3, 2004, pp. 190-195. doi:10.3816/CCC.2004.n.019

[2] C. Shih, V. J. Chen, L. S. Gossett, et al., "LY231514, a Pyrrolo[2,3-d]Pyrimidine-Based Antifolate That Inhibits Multiple Folate-Requiring Enzymes," Cancer Research, Vol. 57, No. 6, 1997, pp. 1116-1123.

[3] C. Cripps, M. Burnell, J. Jolivet, et al., "Phase II Study of First-Line LY231514 (Multi-Targeted Antifolate) in Patients with Locally Advanced or Metastatic Colorectal Cancer: An NCIC Clinical Trials Group Study," Annals of Oncology, Vol. 10, 1999, pp. 1175-1179. doi:10.1023/A:1008372529239

[4] W. John, J. Picus, C. D. Blanke, et al., "Activity of Multitargeted Antifolate (Pemetrexed Disodium, LY231514) in Patients with Advanced Colorectal Carcinoma: Results from a Phase II Study," Cancer, Vol. 88, No. 8, 2000, pp. 1807-1813.

doi:10.1002/(SICI)1097-0142(20000415)88:8<1807::AID -CNCR8>3.0.CO;2-L

[5] C. Underhill, D. Goldstein, V. A. Gorbounova, et al., "A Randomized Phase II Trial of Pemetrexed plus Irinotecan (ALIRI) versus Leucovorin-Modulated 5-FU plus Irinotecan (FOLFIRI) in First-Line Treatment of Locally Advanced or Metastatic Colorectal Cancer," Oncology, Vol. 73, No. 1-2, 2007, pp. 9-20. doi:10.1159/000120626

[6] S. R. Alberts, G. P. Kim, M. R. Mahoney, et al., "Pemetrexed and Oxaliplatin for Metastatic Colorectal Cancer: Results of a Phase I Mayo Cancer Center Research Consortium Trial, MC0248," Clinical Colorectal Cancer, Vol. 6, No. 8, 2007, pp. 572-577. doi:10.3816/CCC.2007.n.024

[7] J. N. Atkins, S. A. Jacobs, H. S. Wieand, et al., "Pemetrexed/Oxaliplatin for First-Line Treatment of Patients with Advanced Colorectal Cancer: A Phase II Trial of the National Surgical Adjuvant Breast and Bowel Project Foundation Research Program," Clinical Colorectal Cancer, Vol. 5, No. 3, 2005, pp. 181-187. doi:10.3816/CCC.2005.n.029

[8] R. Paulson, R. Paulson, J. Hainsworth, C. Geyer, et al., "A Phase II Trial of MTA (Multiple Targeted Antifolate, LY231514) in Patients with 5-FU and Irinotecan-Refractory Colorectal Cancer," American Society of Clinical Oncology, Vol. 18, 1999, 297a.

[9] A. C. McDonald, P. A. Vasey, L. Adams, et al., "A Phase I and Pharmacokinetic Study of LY231514, the Multitargeted Antifolate," Clinical Cancer Research, Vol. 4, No. 3, 1998, pp. 605-610.

[10] D. A. Rinaldi, H. A. Burris, F. A. Dorr, et al., "Initial Phase I Evaluation of the Novel Thymidylate Synthase Inhibitor, LY231514, Using the Modified Continual Reassessment Method for Dose Escalation," Journal of Clinical Oncology, Vol. 13, No. 11, 1995, pp. 2842-2850.

[11] M. Merl, C. Hoimes, T. Pham, et al., "Is There a Palliative Benefit of Gemcitabine plus Fluoropyrimidines in 
Patients with Refractory Colorectal Cancer?" Expert Opinion on Investigational Drugs, Vol. 18, No. 9, 2009, pp. 257-1264. doi:10.1517/13543780903136724

[12] J. L. Tonkinson, J. F. Worzalla, C. H. Teng, et al., "Cell Cycle Modulation by a Multitargeted Antifolate, LY231514, Increases the Cytotoxicity and Antitumor Activity of Gemcitabine in HT29 Colon Carcinoma," Cancer Research, Vol. 59, No. 15, 1999, pp. 3671-3676.

[13] A. Tesei, L. Ricotti, F. De Paola, et al., "In Vitro Schedule-Dependent Interactions between the Multitargeted Antifolate LY231514 and Gemcitabine in Human Colon Adenocarcinoma Cell Lines," Clinical Cancer Research, Vol. 8, No. 1, 2002, pp. 233-239.

[14] T. M. De Pas, F. Toffalorio, C. Catania, et al., "Gemcitabine and Pemetrexed Combination: The Key Role of the Sequence of Drugs Administration," Annals of Oncology, Vol. 20, No. 10, 2009, pp. 1747-1748. doi:10.1093/annonc/mdp385

[15] C. X. Ma, S. Nair, S. Thomas, et al., "Randomized Phase II Trial of Three Schedules of Pemetrexed and Gemcitabine as Front-Line Therapy for Advanced Non-SmallCell Lung Cancer," Journal of Clinical Oncology, Vol. 23, No. 25, 2005, pp. 5929-5937.

\section{doi:10.1200/JCO.2005.13.953}

[16] K. L. Spindler, N. Pallisgaard, I. Vogelius, et al., "Quantitative Cell Free DNA, KRAS and BRAF Mutations in Plasma from Patients with Metastatic Colorectal Cancer during Treatment with Cetuximab and Irinotecan," Clinical Cancer Research, Vol. 18, No. 4, 2012, pp. 1177-185. doi:10.1158/1078-0432.CCR-11-0564

[17] R. Ferrarotto, K. Machado, M. P. Mak, et al., "A Multicenter, Multinational Analysis of Mitomycin C in Refractory Metastatic Colorectal Cancer", European Journal of Cancer, Vol. 48, No. 6, 2012, pp. 820-826. doi:10.1016/j.ejca.2012.01.008

[18] M. G. Fakih, A. Groman, J. McMahon, et al., "A Randomized Phase II Study of Two Doses of Vorinostat in Combination with 5-FU/LV in Patients with Refractory Colorectal Cancer," Cancer Chemotherapy and Pharmacology, Vol. 69, No. 3, 2012, pp. 743-751.

[19] H. Yasui, T. Yoshino, N. Boku, et al., "Retrospective Analysis of S-1 Monotherapy in Patients with Metastatic Colorectal Cancer after Failure to Fluoropyrimidine and Irinotecan or to Fluoropyrimidine, Irinotecan and Oxaliplatin," Japanese Journal of Clinical Oncology, Vol. 39, No. 5, 2009, pp. 315-320. 